\title{
ULTRASHORT PULSE PROPAGATION THROUGH DEPRESSED-CLADDING CHANNEL WAVEGUIDES IN YAG CRYSTAL: SPATIO-TEMPORAL CHARACTERIZATION
}

Marta Morales-Vidala ${ }^{a, *, 1}$, Íñigo J. Sola ${ }^{a}$, Gabriel R. Castillob, Javier R. Vázquez de Aldana $^{\mathrm{a}}$, and Benjamín Alonso ${ }^{\mathrm{a}}$

Applied Physics Department, Aplicaciones del Láser y Fotónica, University of Salamanca. PI. La Merced s/n, Salamanca E-37008, Spaina

Cátedras CONACYT - Centro de Investigaciones en Óptica (CIO), Parque de Investigación e Innovación Tecnológica (PIIT), Alianza Centro 504, Apodaca 66629, Méxicob

\begin{abstract}
Inscription of optical waveguides by direct femtosecond laser irradiation has become a very versatile tool for the development of integrated photonic devices, such as waveguide lasers, frequency converters or photonic lanterns, among many others. The potential application of such devices for the control and manipulation of ultrashort pulses requires the precise knowledge of the temporal distortions that may be induced in the pulse propagation. Currently, research in this topic is scarce, and to our knowledge there is no previous experimental study on the spatio-temporal characterization at the output of waveguides inscribed inside crystals. Here, we have firstly fabricated depressedcladding waveguides with different modal behavior in YAG crystal by direct femtosecond laser irradiation. Then, we implemented an experimental method based on the fiber coupler assisted spectral interferometry technique, that allows obtaining: 1) the temporal dispersion of a pulse at the output of an inscribed waveguide and, 2) full spatio-spectral and spatio-temporal characterization of the output of single-mode and multi-mode waveguides. Our results suggest that the main contribution to the pulse dispersion is due to the material dispersion. Moreover, we found that multimodal waveguides may induce an appreciable inhomogeneity in the temporal features of the pulses that needs to be taken into account in the design of complex devices.
\end{abstract}

Keywords: Ultrafast optics; channeled waveguides; spectral interferometry; phase measurements; pulse measurements

\section{Introduction}

Ultrafast laser inscription ${ }^{2}(\mathrm{ULI})$ is a technique that allows the localized and controlled modification of the refractive index of transparent dielectrics [1], through the non-linear interaction of a femtosecond pulse with the target. The application of such technique to the fabrication of optical waveguides was demonstrated for the first time [2] in 1996, and it has attracted great attention since then due to the possibility to fabricate threedimensional photonic elements with any arbitrary geometry: this 3D processing capability offers a clear advantage of ULI over other conventional microfabrication techniques (i.e. photolithography) that are limited to planar structuring.

In many glasses, with fused silica being the most common example, a refractive index increase can be produced in the region where the femtosecond laser pulse is focused [2]. Then, the fabrication of a waveguide is straightforward by directly writing the channels with an increased index that will constitute the waveguide core. In this approach, the inscription of complex devices and 3D optical circuits [3] is direct and simple, and it has been exploited in different fields such as astrophotonics [4],

\footnotetext{
${ }^{1}$ Present address: I.U. Física Aplicada a las Ciencias y las Tecnologías Universidad de Alicante, Apartado de correos 99, Alicante E-03080, Spain;

${ }^{2}$ ULI
} 
optofluidics [5], telecommunications [6] or bio-sensing [7], among others. In particular, some devices fabricated by ULI in glasses have been successfully employed for ultrafast applications, mainly in supercontinuum generation [8] or in femtosecond pulse generation [9].

Crystalline transparent dielectrics are also desirable substrates for photonic devices integration by ULI due to their excellent optical properties that could improve their functionality, including: high transparency, isotropy or anisotropy, non-linear response, spectroscopic behavior, electro-optical effect. However, a refractive index increase in the focal region can be created only in very few crystals [10] provided that laser irradiation and damage is typically linked with a local amorphization. Then, in most crystals, other strategies must be employed for waveguide photo-inscription, what makes the fabrication of complex devices technically more difficult. The formation of depressedindex claddings $[11,12]$ has emerged as a highly versatile and efficient way to produce photonic guiding elements with arbitrary geometries. In this approach, a large number of parallel low-index laser damage tracks are produced to construct the waveguide cladding, leaving unaffected the waveguide core where the light propagates through. Efficient devices have been fabricated in crystals based on this approach such as waveguide lasers [13], frequency converters [14], beam shapers/splitters [15] or biosensors [16], that operate in the continuous wave or long-pulse regimes. To the authors knowledge, the use of cladding waveguides for ultrafast applications has not been explored yet. However, the features of these waveguides (minimal affection of the core region, shape and size configurable on demand, or isotropic confinement) together with the properties of crystalline materials, make them a very attractive platform for the development of ultrafast devices. For instance, waveguide frequency converters could benefit from the high power achieved at the peak pulse and the non-linear properties of certain crystals, active optical sensors could improve their resolution making use of the ultrashort duration of the pulses, or some complex optical setups (including mirrors, splitters, etc) could be integrated in a single compact device. With this potential in mind, the characterization of the temporal behavior of ultrashort pulses propagating through the waveguides is necessary.

To our knowledge, there is only a previous work [17] in which the temporal dispersion introduced by an ultrafast directly written waveguide fabricated in glass was measured. In that work, the authors studied the temporal dispersion of a pulse propagating through a waveguide inscribed in gallium lanthanum sulphide glass substrate. Their experimental setup was based on white-light spectral-domain interferometry which comprised a midIR supercontinuum source and a Mach-Zehnder free space interferometer. The delay between the test and the reference beams was obtained by Fourier-transform of the interference spectrum.

In our work, we present the spatio-temporal characterization of femtosecond pulses propagating through depressed-cladding waveguides fabricated in yttrium aluminum garnet ${ }^{3}(Y A G)$ crystals by ULI. Different cladding geometries and sizes have been tested, both with monomodal as multimodal behavior, in order to check the potential effect of the cladding structure and the modal behavior in the degradation of the pulse shape. The technique used for the reconstruction of the pulses is based on the fiber coupler assisted spectral interferometry technique ${ }^{4}(\mathrm{STARFISH})[18]$ that uses the Fourier-Transform

\footnotetext{
${ }^{3}$ YAG

${ }^{4}$ STARFISH
} 
Spectral Interferometry ${ }^{5}$ (FTSI) algorithm [19]. It is a versatile, accurate, and simple technique to obtain the spatio-temporal phase of a spatio-temporally complex pulse. Its high temporal and spatial resolutions (femtoseconds and micrometers, respectively) have allowed to obtain complex distribution of light of, for example, diffractive optical elements [20], non-linear effects or few-cycle pulses. The different spatio-temporal structures of pulses propagated to mono-modal and multi-modal waveguides are also presented. We propose the optical circuits based on cladding waveguides in crystals as an excellent architecture for the design of ultrafast devices and pulse control with temporal distortion limited to the substrate dispersion.

\section{Material and methods}

\subsection{Femtosecond laser inscribed waveguide fabrication}

The waveguides studied in this work were inscribed by ULI in a polished YAG crystal cut to the dimensions of $10 \times 20 \times 2 \mathrm{~mm}^{3}$ (Fig. 1a). We have chosen YAG crystal since it is an excellent host material due to its outstanding optical, thermal and mechanical properties. Moreover, it is an isotropic crystal, which is very convenient for our preliminary studies as it reduces the complexity that different group velocities could introduce in the pulse dynamics.

The laser beam used was a Ti:Sapphire ${ }^{6}(\mathrm{Ti}: \mathrm{Sa})$ laser system amplified with a regenerative amplifier (Spitfire, Spectra Physics), emitting femtosecond pulses with a repetition rate of $1 \mathrm{kHz}$ and 100 fs pulses at full width half maximum ${ }^{7}(\mathrm{FWHM})$ at the central wavelength of $\lambda_{0}=795 \mathrm{~nm}$. The pulse energy was reduced by using a set of a half-wave plate and a linear polarizer, followed by a calibrated neutral density filter. With this setup, the energy was reduced to the range of $0.15-0.2 \mu \mathrm{J} /$ pulse in order to fabricate the desired structures.

The laser beam was perpendicularly focused into the sample (100 $\mu \mathrm{m}$ below the sample surface) through the largest faces of the sample using a $50 \times$ microscope objective (numerical aperture ${ }^{8} \mathrm{NA}=0.6$ ). The focus point is controlled by moving the $z$-axis of a high-resolution three-axes motorized stage in which the sample is placed (resolution of $z$-axis: $\pm 1 \mu \mathrm{m}$ ). Firstly, the laser beam is focused on the upper sample surface. To this end, an auxiliary image forming system is used (CMOS camera and objective lens), that simultaneously images the sample surface and the beam focused through the microscope objective. The $z$ position of the stage is finely moved until the optimum focal position is found (minimum spot size). Then, taking this position as reference, the sample is moved up to the desired $z$ position inside the sample. Finally, the sample is moved parallel to the longest edges of the crystal in order to perform a waveguide transverse inscription with the desired geometry. The sample was moved at a constant velocity of $500 \mu \mathrm{m} / \mathrm{s}$ along the $20 \mathrm{~mm}$ long edge and thus parallel damage tracks were inscribed to conform the waveguides with the desired geometry.

\footnotetext{
${ }^{5}$ FTSI

${ }^{6} \mathrm{Ti}: \mathrm{Sa}$

${ }^{7}$ FWHM

${ }^{8} \mathrm{NA}$
} 
Hexagonal photonic lattice-like ${ }^{9}$ (HEX) and circular ${ }^{10}(\mathrm{CIRC})$ depressed-cladding geometries were fabricated, as it is shown in the pictures taken with an optical microscope (Axio Imager, Carl Zeiss) operating in transmission mode in Fig. 1b and 1c.
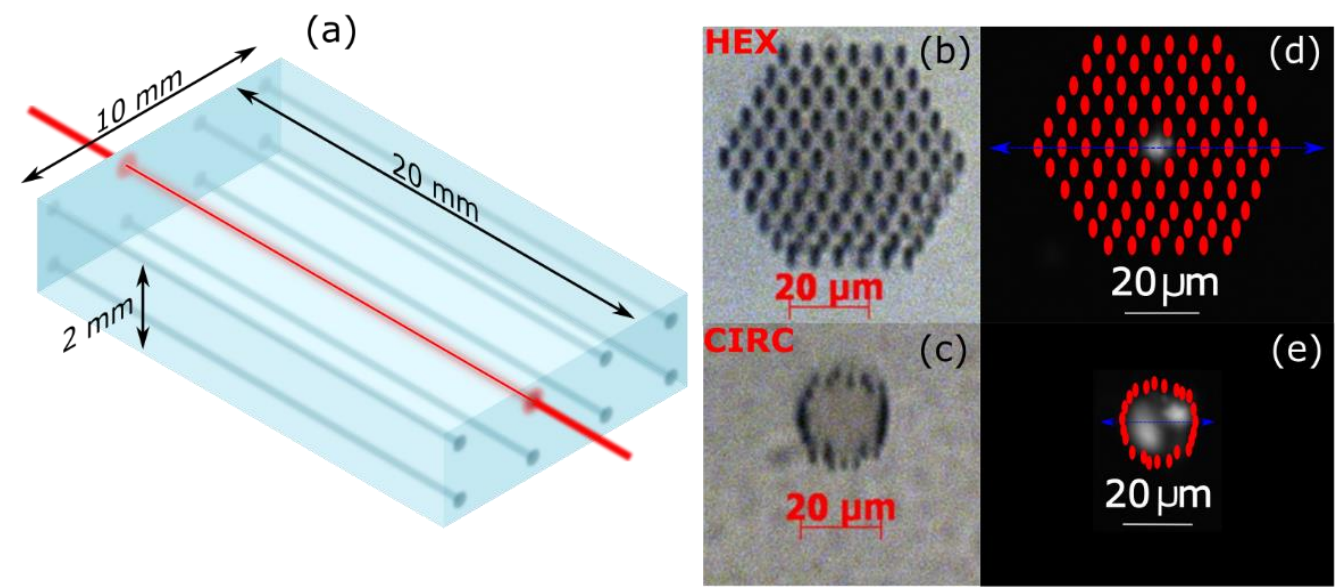

Figure 1.- a) YAG sample sketch with different inscribed waveguides. b) and c) Microscope image of the transversal face of the hexagonal photonic-like waveguide (HEX) and circular cladding waveguide (CIRC). d) and e) waveguide modal output profiles superimposed on the respective waveguide transversal section (red ellipses); blue arrows indicate the scanned direction for the spatio-temporal characterization.

\subsection{Spatio-spectral and spatio-temporal pulse characterization}

Ultrashort laser pulses obtained directly from the femtosecond oscillator (Tsunami, Spectra Physics) at a repetition rate of $80 \mathrm{MHz}$ emitting $100 \mathrm{fs}$ (FWHM) pulses ( $\lambda_{0}=$ $795 \mathrm{~nm}, \Delta \lambda=10 \mathrm{~nm}$ ) were coupled into the waveguides. The modal intensity profile and pulse features were analyzed at the output of the waveguides. The setup (Fig. 2) to inspect the intensity beam distribution of the supported modes and the transverse geometries of the different waveguides at their output face, is based in the end-fire coupling technique [21]. The laser beam was split into two replicas, one of them being focused with a microscope objective $(10 x ; N A=0.22)$ into the transverse section of the waveguide (input face). The output signal was collected by another microscope objective (20x; NA = 0.4) and focused to a CMOS camera (IDS uEye SE). In Fig. 1d and 1e, are represented the waveguide modal profiles at the output of the waveguides superimposed on the respective transversal geometries (both images are captured with the CMOS camera).

\footnotetext{
${ }^{9}$ HEX

${ }^{10} \mathrm{CIRC}$
} 


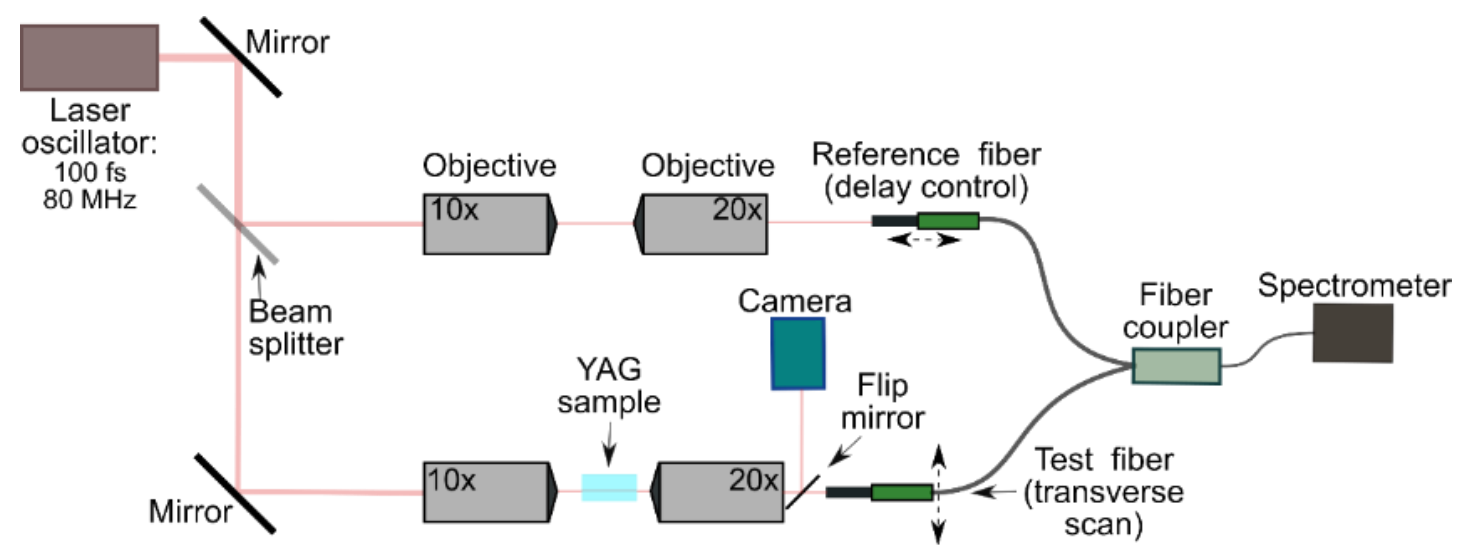

Figure 2.- Experimental setup used for the characterization of the modal intensity profile with the camera (end-fire coupling technique), together with the setup used to obtain the spatio-temporal pulse features at the output of the waveguide (STARFISH technique) by using the fiber coupler.

To characterize the pulse spectral phase acquired after propagating through the waveguide (test signal, unknown), we used spectral interferometry (SI) with the other replica (reference signal, collected by the reference fiber). Both signals were imaged to the two input ports and collected by the single-mode fiber-optic coupler connected to a spectrometer. The reference fiber was similar to the test fiber, in any case, the small difference in dispersion delay was considered by a set-up calibration with spectral interferometry. Then, the delay between the arms is controlled by the position of the reference fiber port along the propagation axis. The relative time delay between the test and reference arms (in the present work it was typically 2 ps) was controlled by using a single axis translation stage parallel to the reference beam. This delay is responsible for the fringes present in the spectral interferences, which encode the relative phase between pulses. The temporal measurement was achieved by extracting the spectral phase difference between the test and the reference pulse. The needed experimental spectra are the individual reference and test pulse, as well as their spectral interferences due to the introduced delay. The test beam signal was collected on axis, as well as spatially scanned (blue arrows, Fig. 1d and 1e) with the corresponding motorized fiber port [18] (STARFISH technique).

It is also important to achieve a similar intensity of light in both replicas (test and reference) to maximize the fringe visibility (the contrast between maxima and minima). We have achieved good contrast by using the same neutral density filter at the reference arm and by using a half-wave plate together with a linear polarizer to attenuate the test arm.

\section{Results}

\section{1.- Pulse temporal profile}

The relative phase difference between the reference and test pulse at a fixed propagation distance is retrieved by using the FTSI algorithm [19], allowing us to obtain the effect introduced by the inscribed waveguide. In Fig. 3a, the red curve shows the spectrum intensity (continuous red curve) and spectral phase of the light after the HEX waveguide acquired on axis (dashed red curve). Previously, we had acquired the SI measurement without any test sample to calibrate the dispersion of the beam splitter and slight differences in fiber lengths at both arms (which could introduce a different amount of dispersion on the pulses). Furthermore, in additional measurements the spectral phase 
introduced by the same amount of YAG substrate (without any fabricated waveguide on the substrate) has also been measured (Fig. 3a, dashed black curve).

The temporal profiles of pulses are obtained by using the corresponding phase, obtained previously by SI, and the spectral amplitude (Fig. 3b). Considering Fourier-limited (77 fs FWHM) input pulses, the temporal FWHM at the output of the HEX waveguide (shown in Fig. $3 \mathrm{~b}$ ) was $113 \pm 1 \mathrm{fs}$, very close to the value obtained at the output of the YAG substrate (104 $\pm 1 \mathrm{fs})$. The slight differences shown between the spectral phases of the pulses (Fig. 3a), together with the comparison of the temporal FWHM of the pulses at the output of the waveguide and at the output of the YAG sample, suggest that the main dispersion contribution can be attributed to the material of the YAG crystal substrate. Therefore, these results denote that the cladding waveguide structure do not add any substantial modification to the input pulses considered in our experiments, which match with a previous study performed in inscribed waveguides by Demetriou et al. [17]. By using a different experimental method and mathematical analysis, they concluded that the main dispersion contribution in the directly written waveguides in sulphide glass is attributed to the material of the substrate. However, as far as we know, the phase of femtosecond pulses at the output of cladding waveguides, has not been previously characterized.
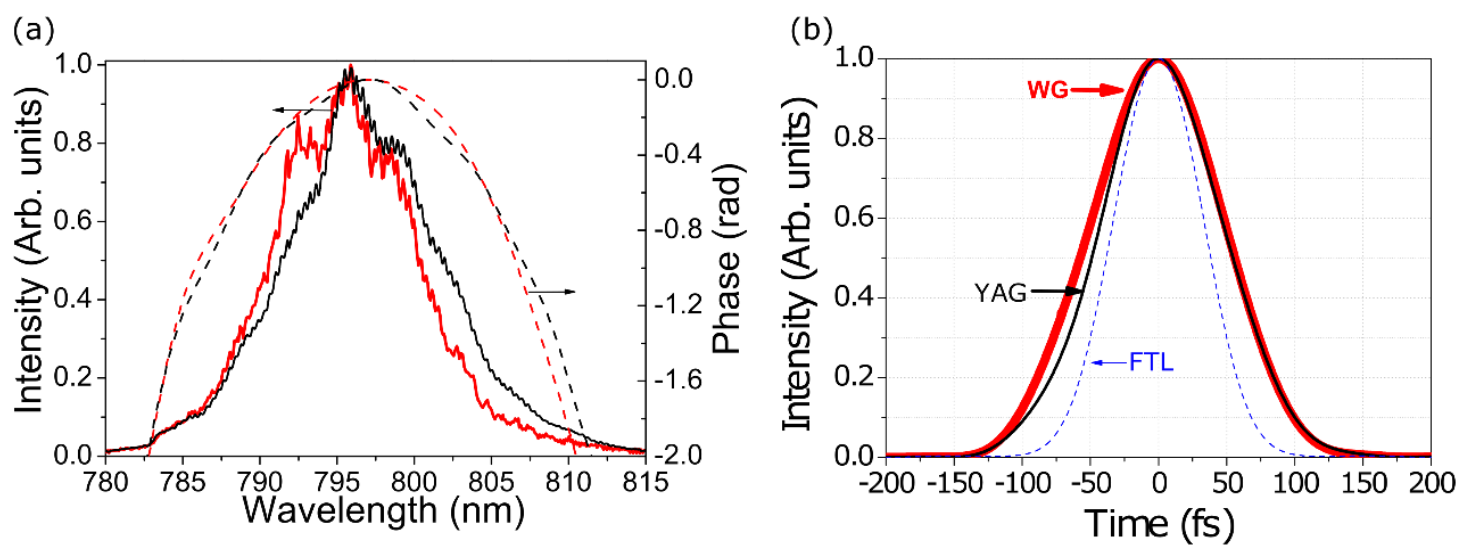

Figure 3.- a) Output spectrum (left axis, continuous curves) and their corresponding spectral phase (right axis, dashed curves) of the pulse at the output of inscribed HEX waveguide (red curves) and of bulk YAG crystal (black curves). b) Output temporal intensity retrieved pulse after propagation through: HEX (WG) waveguide (thick red curve), fresh YAG (thin black curve), and Fourier-limited input (FTL) pulse (dashed blue curve).

A parameter commonly used to evaluate the second order dispersion of a pulse is the Group Delay Dispersion (GDD). The GDD evaluates the accumulated Group Velocity Dispersion $\left(G V D=\left[d_{\omega}^{2} k(\omega)\right]_{\omega=\omega_{0}}\right)$ through a medium with length $L$, i.e. $G D D=G V D \times$ $L$. Dispersive materials have frequency $(\omega)$-dependent refractive indices $n(\omega)$, being the wave number $k(\omega)=n(\omega) \omega / c$. Considering the Sellmeier coefficients for undoped YAG [22] to obtain $n(\omega)$ and the relations indicated above, it has been obtained the theoretical $G D D_{\text {th }}=1986 \mathrm{fs}^{2}$ introduced by $20 \mathrm{~mm}$ of this material at $\lambda=795 \mathrm{~nm}$,. On the other hand, the GDD has been experimentally obtained by fitting the slope of the first spectral phase derivative of the corresponding YAG spectral phases at the spectral interval 785$809 \mathrm{~nm}$. The average GDD in YAG obtained from the 32 fits was $1990 \mathrm{fs}^{2}( \pm 2 \%)$. This comparison between the theoretical and experimental $G D D$ value of the YAG substrate reinforces the reliability and applicability of the method presented in this work. Finally, 
from the analysis of the spectral phase after the waveguide, we have found that the dispersion is close to that of the YAG substrate, therefore concluding that the waveguide has a minor contribution to the pulse dispersion.

\section{2.- Spatio-temporal measurements}

An extension of the method used in the previous section has been performed and it is presented in this work. This method obtains not only the spectral phase at the axis of the test arm, but also at different transversal positions of the waveguide output plane (see Fig. 2, top axis) by scanning different horizontal positions with the test fiber at the STARFISH detection fiber plane (Fig.2, bottom axis). As it was explained in Section 2.2, the second objective of the test arm provides the image of the transversal surface of the substrate (at the output of the waveguide). As the reference fiber is static at a single transversal coordinate, the test beam is always referred to the same pulse and hence the connection between different horizontal positions in transverse profile test can be obtained. The spatial resolution in the plane of the STARFISH fiber is given by the modefield diameter of the single-mode fiber: $4 \mu \mathrm{m}$ [18], whereas taking into account that the magnification factor in this plane is around $28 \mathrm{x}$, the spatial resolution in the plane of ULI waveguides output is $0.15 \pm 0.02 \mu \mathrm{m}$.

Symmetric and mono-modal light distribution coming from a HEX waveguide (Fig. 4a) has been compared to asymmetric and multi-modal spatio-temporal light distribution at the output of a CIRC waveguide (Fig. 4b). The distribution of intensities within the mode profiles at the output of the waveguides was imaged to the camera. Their size can be estimated by the superposition with the geometry structure (real size estimated from the microscope images, see Fig. 1b-e). The spectral (spatio-spectral traces) and temporal features (spatio-temporal reconstructions) of the pulses at the output of mono-modal (HEX) and multi-modal (CIRC) waveguides (shown in Fig. 4) are obtained as a function of the different transversal positions ( $x$ coordinate, indicated by a blue arrow in Fig. 1d and $1 \mathrm{e}$ ) with respect to wavelength or time, respectively.

In Fig. 4a, the spatio-spectral trace of the HEX waveguide is shown. The top axis of the figure indicates the real size dimensions of the transversal waveguide face (at the output ULI waveguides plane). The bottom axis corresponds to the scanning fiber positions after the microscope objective (at the STARFISH detection fiber plane). In this spatial scan, the beam profile was sampled with $10 \mu \mathrm{m}$ step. The spectra are centered at $795 \mathrm{~nm}$ and the spatial mode dimensions at half-maximum is around $6 \mu \mathrm{m}$ (given at the waveguide output). The temporal intensity of the pulse after the HEX waveguide obtained on axis is $\mathrm{FWHM}_{(\mathrm{x}=0)}=115 \pm 1$ fs (as the results discussed in section 3.1). The spatio-temporal intensity distribution (Fig. 4b) does not present deviations, corresponding with a homogeneous beam after the HEX waveguide propagation. In Fig. 5 we analyze in detail the homogeneity of the beam. In the spectral domain, we find that the central wavelength (Fig. 5a) and the bandwidth (Fig. 5b) are practically constant within the mode profile (shown in grey). In the temporal domain, the pulse duration (Fig. $5 \mathrm{c}$ ) is also preserved for each point of the waveguide mode. 
(a)

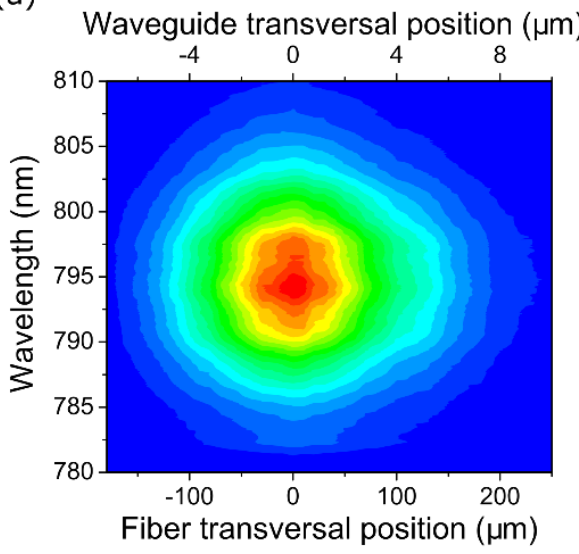

(b)

Waveguide transversal position $(\mu \mathrm{m})$

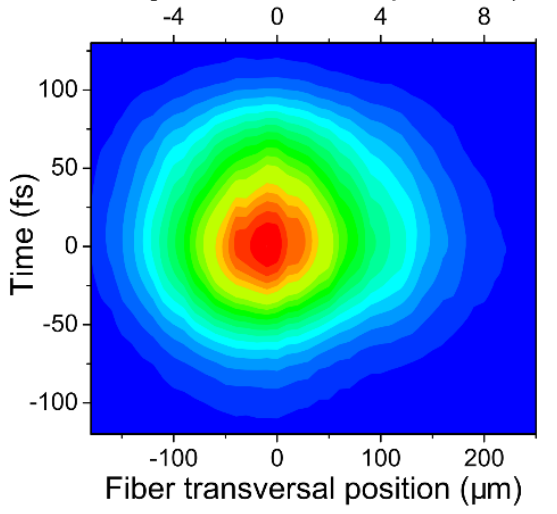

(c)
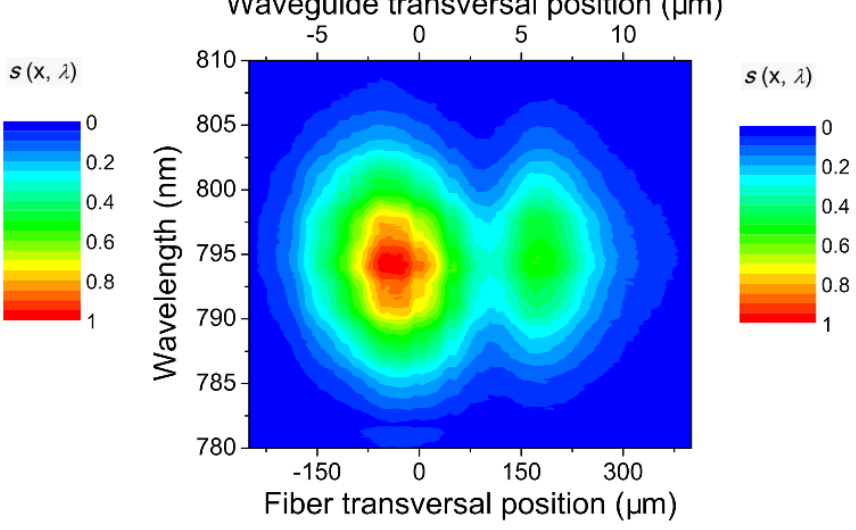

(d)

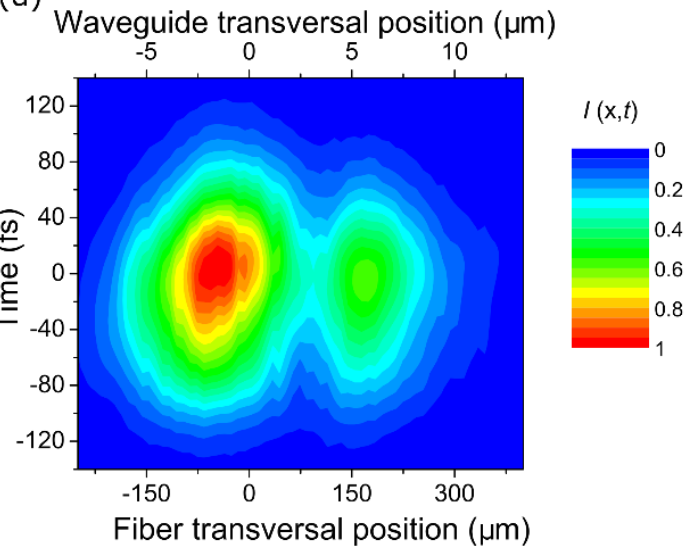

Figure 4.- Experimental spatio-spectral traces at the output of waveguides: a) HEX and c) CIRC waveguides as function of fiber transversal position (bottom axis) and waveguide transversal position (top axis). Spatio-temporal intensity reconstructions of: b) HEX and d) CIRC waveguides as a function of the same fiber and waveguide transversal positions.

Finally, the STARFISH method has been applied to an asymmetric and multimodal light distribution observed at the output of the CIRC waveguide (Fig. 4c-d). The same 10- $\mu \mathrm{m}$ step was used in this case to scan the profile with the collecting fiber. In this waveguide, two peaks separated by $7 \mu \mathrm{m}$ are observed in the intensity modal profile at the waveguide output plane. The peak on the left is slightly bigger in size (FWHM $=6 \mu \mathrm{m}$ ) and energy, while the peak on the right has a width of FWHM $=5 \mu \mathrm{m}$ (Fig. 4c). The temporal features at the maximum intensity of the left distribution is $F W H M=122 \pm 1 \mathrm{fs}$ while at the right mode is $110 \pm 1 \mathrm{fs}$ (Fig. 4d). When analyzing the homogeneity of the beam, we find that the central wavelength (Fig. $5 \mathrm{~d}$ ) is not constant within the left-hand peak and it is slightly different to the right-hand one. We also find more variation in the spectral bandwidth of the CIRC waveguide (Fig. 5e) than that of the HEX waveguide (Fig. 5b). These differences are translated into the temporal domain (Fig. 5f) and, as said before, the peak on the left presents longer pulse duration (with relatively high variation along the transverse position) than the peak on the right. Therefore, we can conclude that the CIRC waveguide clearly presents an inhomogeneity that could be attributed to the multimodal behavior of the waveguide, in comparison to the HEX waveguide that we found to be reasonably homogeneous. Despite these differences, there is not a dramatic temporal or spectral distortion within the beam profile. 

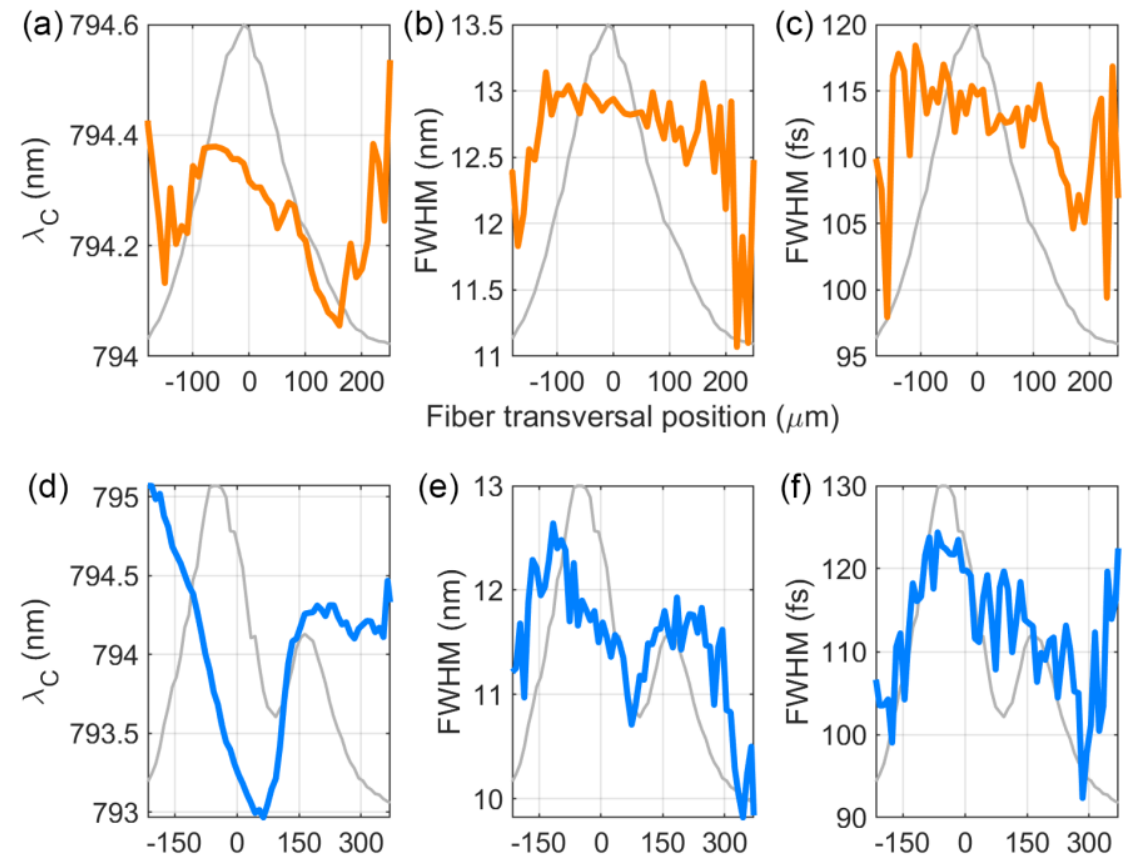

Fiber transversal position $(\mu \mathrm{m})$

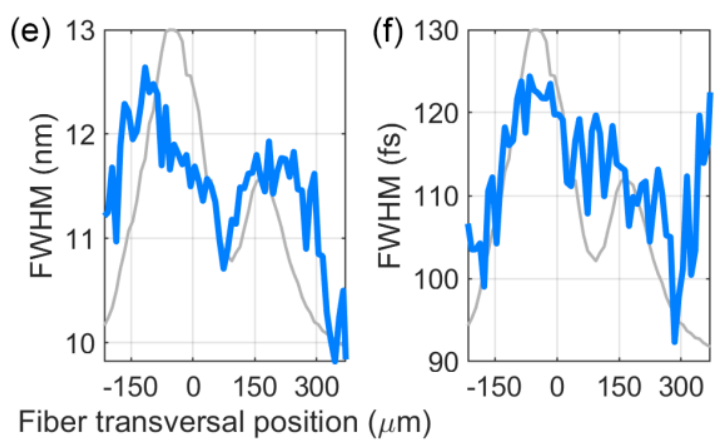

Figure 5.- Experimental details for the modes at the output of waveguides: a) HEX and d) CIRC central wavelength, b) HEX and e) CIRC spectral FWHM, c) HEX and f) CIRC temporal FWHM. In grey it is represented the integrated spatial distribution of energy in the scanned line. All magnitudes are plotted as a function of the fiber transversal position.

\section{Conclusions}

A robust and accurate technique (STARFISH), which provides the spatio-temporal reconstruction of ultrashort pulsed beams, has been used to study the effect of the propagation of femtosecond pulsed beams in different laser inscribed waveguides at their output. Different depressed-cladding channel waveguides were fabricated in YAG crystal (with circular geometry and hexagonal lattice) by femtosecond laser irradiation, and the technique was used to obtain the temporal pulse dispersion introduced in the propagation through them. In the range of experimental parameters used here, we have shown that the greatest dispersive effect comes from the material, with small alterations due to the geometry of the waveguide. This is the first study, to our knowledge, related with the dispersion effect produced by depressed-cladding channel waveguides. We also show, for the first time, the spatio-spectral and spatio-temporal structure of pulsed beams at the output of different inscribed waveguides. We have obtained that symmetric monomodal distributions (hexagonal cladding waveguide) are homogeneous in comparison with asymmetric multimodal distributions (circular cladding waveguide), which present moderate spectral and temporal differences along the beam profile. These spectral and temporal distortions should be carefully taken into account in the design of integrated photonic devices for ultrashort pulse manipulation.

Acknowledgments: This work was supported by Junta de Castilla y León (Project SA046U16 and SA287P18) and MINECO (Projects FIS2017-87970-R, EQC2018004117-P). M.M.-V. acknowledges support from Junta de Castilla y León grant (No. SA046U16) and from Generalitat Valenciana, Spain, under project CDEIGENT/2018/024. 


\section{References}

[1] R.R. Gattass, E. Mazur, Femtosecond laser micromachining in transparent materials, Nat. Photonics. 2 (2008) 219-225.

[2] K.M. Davis, K. Miura, N. Sugimoto, K. Hirao, Writing waveguides in glass with a femtosecond laser, Opt. Lett. 21 (1996) 1729-1731.

[3] R. Osellame, G. Cerullo, R. Ramponi, Femtosecond Laser Micromachining, Springer-Verlag Berlin Heidelberg, 2012.

[4] R.R. Thomson, A.K. Kar, J. Allington-Smith, Ultrafast laser inscription: an enabling technology for astrophotonics, Opt. Express. 17 (2009) 1963.

[5] A. Crespi, Y. Gu, B. Ngamsom, H.J.W.M. Hoekstra, C. Dongre, M. Pollnau, R. Ramponi, H.H. Van Den Vlekkert, P. Watts, G. Cerullo, R. Osellame, Threedimensional Mach-Zehnder interferometer in a microfluidic chip for spatiallyresolved label-free detection, Lab Chip. 10 (2010) 1167-1173.

[6] R.R. Thomson, H.T. Bookey, N.D. Psaila, A. Fender, S. Campbell, W.N. MacPherson, J.S. Barton, D.T. Reid, A.K. Kar, Ultrafast-laser inscription of a three dimensional fan-out device for multicore fiber coupling applications, Opt. Express. 15 (2007) 11691-11697.

[7] R.W. Applegate, J. Squier, T. Vestad, J. Oakey, D.W.M. Marr, P. Bado, M.A. Dugan, A.A. Said, Microfluidic sorting system based on optical waveguide integration and diode laser bar trapping, Lab Chip. 6 (2006) 422-426.

[8] N.D. Psaila, R.R. Thomson, H.T. Bookey, S. Shen, N. Chiodo, R. Osellame, G. Cerullo, A. Jha, A.K. Kar, Waveguide fabrication and supercontinuum generation in an ultrafast laser inscribed chalcogenide glass waveguide, Opt. Express. 15 (2007) 15776-15781.

[9] S.J. Beecher, R.R. Thomson, N.D. Psaila, Z. Sun, T. Hasan, A.G. Rozhin, A.C. Ferrari, A.K. Kar, 320 fs pulse generation from an ultrafast laser inscribed waveguide laser mode-locked by a nanotube saturable absorber, Appl. Phys. Lett. 97 (2010) 111114.

[10] F. Chen, J.R.V. de Aldana, Optical waveguides in crystalline dielectric materials produced by femtosecond-laser micromachining, Laser Photonics Rev. 8 (2014) 251-275.

[11] A.G. Okhrimchuk, A. V Shestakov, I. Khrushchev, J. Mitchell, Lipp-Project-Report, 30 (2005) 2248-2250.

[12] D.G. Lancaster, S. Gross, H. Ebendorff-Heidepriem, K. Kuan, T.M. Monro, M. Ams, A. Fuerbach, M.J. Withford, Fifty percent internal slope efficiency femtosecond direct-written $\mathrm{Tm}^{3+}:$ ZBLAN waveguide laser, Opt. Lett. 36 (2011) 1587-1589.

[13] A. Okhrimchuk, V. Mezentsev, A. Shestakov, I. Bennion, Low loss depressed cladding waveguide inscribed in YAG: Nd single crystal by femtosecond laser pulses, Opt. Express. 20 (2012) 3832-3843.

[14] Y. Jia, J.R. V. de Aldana, C. Romero, Y. Ren, Q. Lu, F. Chen, FemtosecondLaser-Inscribed BiB3O6 Nonlinear Cladding Waveguide for Second-Harmonic Generation, Appl. Phys. Express. 5 (2012) 72701.

[15] Y. Jia, C. Cheng, J.R. Vázquez De Aldana, G.R. Castillo, B. Del Rosal Rabes, Y. Tan, D. Jaque, F. Chen, Monolithic crystalline cladding microstructures for 
efficient light guiding and beam manipulation in passive and active regimes, Sci. Rep. 4 (2014) 5988.

[16] G. Li, H. Li, R. Gong, Y. Tan, J. Rodríguez Vázquez de Aldana, Y. Sun, F. Chen, Intracavity biosensor based on the Nd:YAG waveguide laser: tumor cells and dextrose solutions, Photonics Res. 5 (2017) 728-732.

[17] G. Demetriou, J.-P. Bérubé, R. Vallée, Y. Messaddeq, C.R. Petersen, D. Jain, O. Bang, C. Craig, D.W. Hewak, A.K. Kar, Refractive index and dispersion control of ultrafast laser inscribed waveguides in gallium lanthanum sulphide for near and mid-infrared applications, Opt. Express 24 (2016) 6350.

[18] B. Alonso, Í.J. Sola, Ó. Varela, J. Hernández-Toro, C. Méndez, J. San Román, A. Zaïr, L. Roso, Spatiotemporal amplitude-and-phase reconstruction by Fouriertransform of interference spectra of high-complex-beams, J. Opt. Soc. Am. B. 27 (2010) 933-940.

[19] L. Lepetit, G. Cheriaux, M. Joffre, Linear techniques of phase measurement by femtosecond spectral interferometry for applications in spectroscopy, J. Opt. Soc. Am. B 12 (1995) 2467-2474.

[20] O. Mendoza-Yero, B. Alonso, G. Mínguez-Vega, Í.J. Sola, J. Lancis, J.A. Monsoriu, Synthesis of fractal light pulses by quasi-direct space-to-time pulse shaping, Opt. Lett. 37 (2012) 1145-1147.

[21] J.T. Boyd, D.B. Anderson, Electronics Research Division, Radiation pattern of an end-fire optical waveguide coupler, Opt. Comm. 13 (1975) 353-358.

[22] D.E. Zelmon, D.L. Small, R. Page, Refractive-index measurements of undoped yttrium aluminum garnet from 0.4 to $5.0 \mu \mathrm{m}$, Appl. Optics. 37 (1998) 4933. 